\title{
A teaching-learning module on stellar structure and evolution
}

\author{
Silvia Galano ${ }^{1}$, Arturo Colantonio ${ }^{2}$, Silvio Leccia ${ }^{3},{\text { Emanuella } \text { Puddu }^{4}, \text { Irene Marzoli }^{1} \text { and }}$ \\ Italo Testa ${ }^{5}$ \\ ${ }^{1}$ Physics Division, School of Science and Technology, University of Camerino, Via Madonna delle \\ Carceri 9, I-62032 Camerino (MC), Italy \\ ${ }^{2}$ Liceo Scientifico e delle Scienze Umane "Cantone", Via Savona, I-80014 Pomigliano d'Arco, Italy \\ ${ }^{3}$ Liceo Statale "Cartesio", Via Selva Piccola 147, I-80014 Giugliano, Italy \\ ${ }^{4}$ INAF - Capodimonte Astronomical Observatory of Naples, Salita Moiariello, 16, I-80131 Napoli, \\ Italy \\ ${ }^{5}$ Department of Physics "E. Pancini", "Federico II" University of Napoli, Complesso Monte \\ S.Angelo, Via Cintia, I-80126 Napoli, Italy
}

\begin{abstract}
We present a teaching module focused on stellar structure, functioning and evolution. Drawing from literature in astronomy education, we identified three key ideas which are fundamental in understanding stars' functioning: spectral analysis, mechanical and thermal equilibrium, energy and nuclear reactions. The module is divided into four phases, in which the above key ideas and the physical mechanisms involved in stars' functioning are gradually introduced. The activities combine previously learned laws in mechanics, thermodynamics, and electromagnetism, in order to get a complete picture of processes occurring in stars. The module was piloted with two intact classes of secondary school students $(\mathrm{N}=59$ students, $17-18$ years old) and its efficacy in addressing students' misconceptions and wrong ideas was tested using a ten-question multiple choice questionnaire. Results support the effectiveness of the proposed activities. Implications for the teaching of advanced physics topics using stars as a fruitful context are briefly discussed.
\end{abstract}

\section{Introduction}

We present a teaching module (TM) focused on stars, as they are the fundamental constituents of the Universe. Stars can be an exceptionally motivating context to teach cross-concepts in science as energy, equilibrium or the properties of matter, and hence deserved a dedicated module. In particular teaching about basic functioning and properties of stars may help students deepen knowledge about forces, hydrostatics, thermodynamics, nuclear reactions, light emission and propagation, and understand how all these content areas are related to each other. Moreover, understanding stars' inner processes can help students to: (i) develop a scientifically informed knowledge on how chemical elements are created - nuclear fusion of light elements (hydrogen and helium) into heavier ones (including, e.g., carbon, oxygen, and iron); (ii) justify chemical abundances in the Universe; (iii) understand conditions under which life may develop. Previous studies were focused primarily on fostering students' understanding about celestial motion, seasons and Moon phases [1-4], while no study until now described the development of research-based teaching-learning activities about stars. This paper aims at addressing this issue by blending

1 Corresponding author: silvia.galano@unicam.it 
in an innovative way paper-and-pencil tasks with laboratory activities exploiting Tracker $^{2}$ and data-logging spectrometer analysis.

\section{Issue in teaching about the stars}

Research into students' understanding about stars is rather limited [5] and is focused primarily on investigating students' misconceptions about stars. Earlier studies [6] show that students often do not distinguish between stars and planets and think that the Milky Way is composed by stars that are very close each other. In other cases, some students think that the greater the radius of a Star is, the bigger its mass gets, thus disregarding the mechanisms behind evolution of the stars. Students are not able to estimate distances between stars and Earth and often do not understand that stars are not motionless celestial objects. Results from more recent studies [7] show that students think that a star is a "burning object", which releases some kind of "energy", thus confusing chemical and burning reactions with nuclear reactions, which are the responsible for stars' inner physical processes (expansion, compression, temperature variations). Many students have difficulties in describing stars' light emission and think they emit monochromatic light. Furthermore a lot of students interpret the Hertzsprung-Russell (H-R) diagram as a trajectory or a position vs. time graph. Students rarely explain stars formation and functioning in a detailed way. For instance, Bailey [8, 9] showed that few students correctly recognize the role of gravity, the central force in the star formation process. Similarly, only few students recognize the role of temperature in the stars' inner processes.

From the above literature, it emerges that students' difficulties in understanding stars formation and functioning are mainly related to a scarce knowledge of the underlying physics mechanisms. In particular, we grouped students' conceptions in three distinct categories: (i) conceptions about the forces involved in stars' formation and equilibrium; (ii) conceptions about stars' emitted radiation; (iii) conceptions about stars' inner processes. Therefore we identified three key ideas around which content knowledge about stars can be reconstructed: (i) mechanical and thermal equilibrium; (ii) spectral analysis; (iii) energy and nuclear reactions. Building on these key ideas, differently from traditional proposals in astronomy education ${ }^{3}$, the students learn how to link together important areas in physics as classical mechanics/thermodynamics, electromagnetism and modern physics through simple experiments and modelling tasks.

\section{Activities of the module}

The module is divided into four phases, in which the key ideas for describing stars' functioning and physical mechanisms are gradually introduced. Table 1 summarizes the activities, the objectives and the key ideas related to each TM phase.

Table 1: Overview of the "Stars and their functioning" student's activities.

\begin{tabular}{|c|c|c|c|c|c|}
\hline Phase & Key ideas & $\begin{array}{c}\text { Time } \\
\text { (h) }\end{array}$ & Intended objectives & Activities - What students do & $\begin{array}{l}\text { Teaching } \\
\text { materials } \\
\text { and } \\
\text { resources }\end{array}$ \\
\hline
\end{tabular}

\footnotetext{
2 Tracker is a free video analysis and modeling tool built on the (OSP) Java framework. It is designed to be used in physics education (https://physlets.org/tracker/)

3 Example of teaching activities about stars can be found at http://www.lpi.usra.edu/education/pre_service_edu/StellarActivities.shtml or http://imagine.gsfc.nasa.gov/educators/lifecycles/Imagine2.pdf
} 


\begin{tabular}{|c|c|c|c|c|c|}
\hline 1 & $\begin{array}{l}\text { Introduction } \\
\text { to stars' } \\
\text { parameters }\end{array}$ & 2 & $\begin{array}{l}\text { To identify stellar } \\
\text { quantities that can be } \\
\text { measured }\end{array}$ & $\begin{array}{l}\text { Discuss in small groups using } \\
\text { sketches and words } \\
\text { Determine Sun's mass through } \\
\text { Newton's law }\end{array}$ & Worksheet \\
\hline \multirow{4}{*}{2} & \multirow{4}{*}{$\begin{array}{l}\text { Spectral } \\
\text { analysis }\end{array}$} & \multirow{4}{*}{8} & $\begin{array}{l}\text { To infer information } \\
\text { about stars' } \\
\text { composition and } \\
\text { processes from } \\
\text { spectra }\end{array}$ & $\begin{array}{l}\text { Estimate the fundamental } \\
\text { frequency of Sun pressure } \\
\text { waves } \\
\text { Estimate Sun's radius using the } \\
\text { equation for the fundamental } \\
\text { frequency of Sun pressure } \\
\text { waves }\end{array}$ & $\begin{array}{l}\text { Software: } \\
\text { Goldwave }\end{array}$ \\
\hline & & & $\begin{array}{l}\text { different physical } \\
\text { processes from } \\
\text { spectral graphs }\end{array}$ & $\begin{array}{l}\text { Discuss the differences between } \\
\text { spectra of fluorescent and } \\
\text { incandescent lamps }\end{array}$ & $\begin{array}{l}\text { Software: } \\
\text { Spectralab }\end{array}$ \\
\hline & & & \multirow{2}{*}{$\begin{array}{l}\text { To establish a } \\
\text { relationship between } \\
\text { emitted light and Sun } \\
\text { surface temperature }\end{array}$} & $\begin{array}{l}\text { Analyse the Sunlight spectrum } \\
\text { and discuss Planck's blackbody } \\
\text { radiation function }\end{array}$ & $\begin{array}{l}\text { Software: } \\
\text { Spectralab }\end{array}$ \\
\hline & & & & $\begin{array}{l}\text { Determine Sun's surface } \\
\text { temperature through Wien's law }\end{array}$ & $\begin{array}{l}\text { Software: } \\
\text { Logger Pro }\end{array}$ \\
\hline \multirow[b]{2}{*}{3} & \multirow[b]{2}{*}{$\begin{array}{l}\text { Mechanical } \\
\text { and thermal } \\
\text { equilibrium }\end{array}$} & \multirow[b]{2}{*}{6} & \multirow{2}{*}{$\begin{array}{l}\text { To introduce the role } \\
\text { of gravitational force } \\
\text { in the stars' } \\
\text { functioning } \\
\text { mechanism } \\
\text { To justify the need } \\
\text { for pressure forces }\end{array}$} & $\begin{array}{l}\text { Estimate forces acting on a } \\
\text { Sun's volume element }\end{array}$ & Worksheet \\
\hline & & & & Estimate Sun's rotational speed & $\begin{array}{l}\text { Software: } \\
\text { Tracker }\end{array}$ \\
\hline 4 & $\begin{array}{l}\text { Energy and } \\
\text { nuclear } \\
\text { reactions }\end{array}$ & 4 & $\begin{array}{l}\text { To justify stars' } \\
\text { functioning with } \\
\text { increasing production } \\
\text { of energy and of } \\
\text { chemical elements } \\
\text { To understand that } \\
\text { evolution of a star } \\
\text { depends only on its } \\
\text { initial quantity of } \\
\text { mass }\end{array}$ & $\begin{array}{l}\text { Estimate energy delivered by } \\
\text { the Sun } \\
\text { Discuss basic nuclear reactions } \\
\text { inside the Sun } \\
\text { To distinguish between } \\
\text { chemical and nuclear reactions }\end{array}$ & Worksheet \\
\hline
\end{tabular}

Corresponding driving questions are "What is a star?", "Which physical quantities would you use to describe the stars' functioning?" "How can we measure such quantities?" (Phase 1) "Which quantities can we measure using light emitted by stars?" (Phase 2), "What is the shape of a star and why?", "How can a star be in equilibrium?" (Phase 3), "How you think a star is functioning?" (Phase 4). Table 1 summarizes the proposed students' activities.

\subsection{First phase: introduction to stars' parameters}

Students are involved in Inquiry Based activities and are introduced to the study of stars according to the following driving questions: "What is a star?", "Which physical quantities would you use to describe the stars' functioning?" "How can we measure such quantities?". During a guided discussion students are driven to identify the stellar quantities that will be studied throughout the module: (i) radius, (ii) mass, (iii) temperature and (iv) matter composition. For the aims of the module, we will rely on the Sun, because it is a typical star in the main sequence of the H-R diagram; moreover it is the closest star and its distance 
from Earth $\left(1.5 \times 10^{8} \mathrm{~km}\right)$ is taken as the reference for astronomical distances in the Solar System.

\subsection{Second phase: spectral analysis}

We introduce the second phase activities asking students the following driving questions "Which quantities can we measure using light emitted by stars?". The discussion aims at reinforcing students' knowledge about waves and at relating spectrum analysis with the Sun's parameters. The activity presented by Leccia et al. [10] may be a useful starting point. Students are guided to recognise that pressure waves are generated in inner shells of the Sun due to internal processes and that the fundamental frequency of such oscillations is related to Sun's geometrical factors, the radius, temperature and mass. Since the solar mass has been calculated in the previous phase, students' attention is here focused on the remaining two parameters, radius and temperature, one of which has to be determined with an independent measurement.

To cope with this issue, we propose to study light spectra. Students are involved in inquiry based activities and learn how they can use light spectra to gain knowledge about the internal structure/processes of an inaccessible source, as the Sun.

Students first investigate spectra obtained from a fluorescent and a filament lamp (equivalent in power) using a data logging spectrometer (Leybold Spectralab ${ }^{4}$ ). Drawing on the empirical evidence that the filament lamp warms up much more than the fluorescent one, students can understand that different physical processes - thermal and stimulated emission - happen in the two sources, and that such differences are reflected in the continuous versus discrete appearance of the two curves. We let students observe spectra acquired from Sodium and Cadmium bulbs, to show that light spectrum depends also on the chemical elements involved in the emission process, and that the interaction between light and a chemical element may affect the original spectrum causing absorption lines.

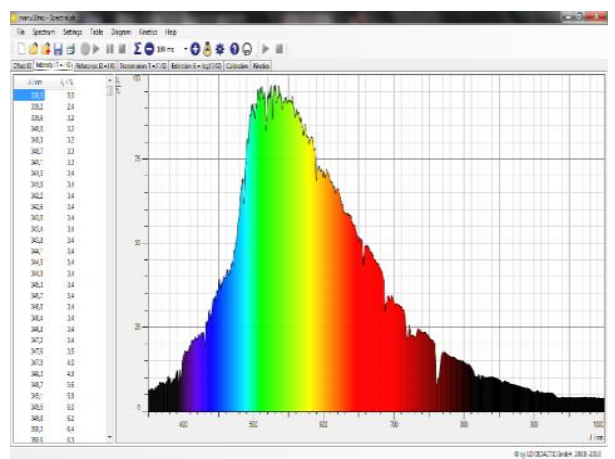

Fig. 1. Non calibrated spectrum curve of the Sun light measured from outside a window of the classroom with Leybold spectrometer and Spectralab software.

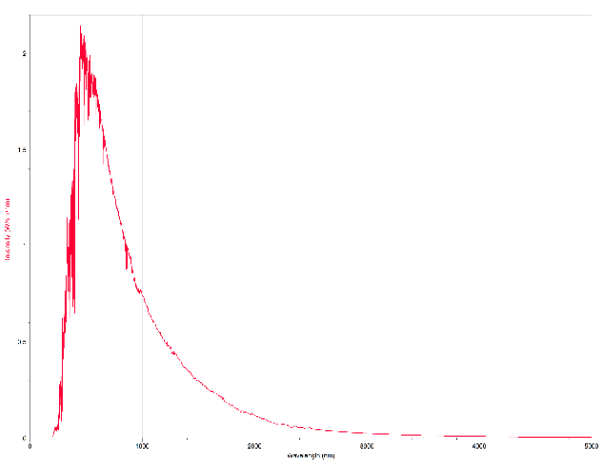

Fig. 2. Spectrum curve of the Sun light measured from space (source: http://vaiitk.vlabs.ac.in/images/expt4/lines.png). Data are expressed in $W /\left(\mathrm{m}^{3}\right) 10^{9}$.

Students are then asked to investigate the Sun spectrum measured from outside a window of the classroom using the Leybold spectrometer. They find out that this spectrum is a continuous one, due to a thermal emission process (Fig. 1). To investigate solar

\footnotetext{
${ }^{4}$ http://www.leybold-shop.com/physics/physics-equipment 89/optics/spectroscopy/spectrophotometer/spectralab467250.html
} 
spectrum without interference of Earth's atmosphere, students plot available data ${ }^{5}$ and analyse the resulting graph (Fig. 2). Building on the similarities with the incandescent bulb spectrum, students can understand that the main physical quantity that can be determined from the Sun's spectrum is the temperature of its surface. At this point, the blackbody Planck function and Wien's law can be introduced to quantitatively determine the temperature of the Sun's surface.

Finally, students' attention is focused on the minima of the spectra measured from outside the window of their classroom (Fig. 1) and from the space (Fig. 2). Since they represent missing points in the plot, they can be related to some process of absorption by chemical elements in Sun's or Earth's atmosphere. Comparing the two spectra, students should be able to understand that in the spectrum measured from outside their window they can find absorption lines due to molecules (e.g. $O^{2}, 780 \mathrm{~nm}$, see Fig. 1). In the spectrum measured from space, absorption lines are due only to chemical elements (e.g. $H$ ) belonging to photosphere of the Sun.

\subsection{Third phase: mechanical and thermal equilibrium}

Starting from driving questions such as "What is the shape of a star and why?", "How can a star be in equilibrium?" we introduce the study of stars' equilibrium. The students are first guided to understand that the spherical symmetry of the gravitational force determines the spherical shape of the stars. To investigate why stars do not collapse, students are then shown a video of sunspots at equatorial latitude, from which they can infer that the Sun (and, more generally, stars) rotates. Similarly to recent teaching proposals $[11,12]$, students use Tracker to measure the Sun's rotational velocity (Fig. 3). Distinct from these proposals, here students apply Newton's law to determine numerically the centripetal acceleration and the gravitational acceleration of the Sun, and infer that other forces must essentially balance gravitational force.

At this point, students are asked to hypothesize what other forces act on the volume element. By recalling buoyancy acting on every object in a fluid, students can understand that such other forces are due to pressure forces acting on the surface of the volume element as in the case of liquids.
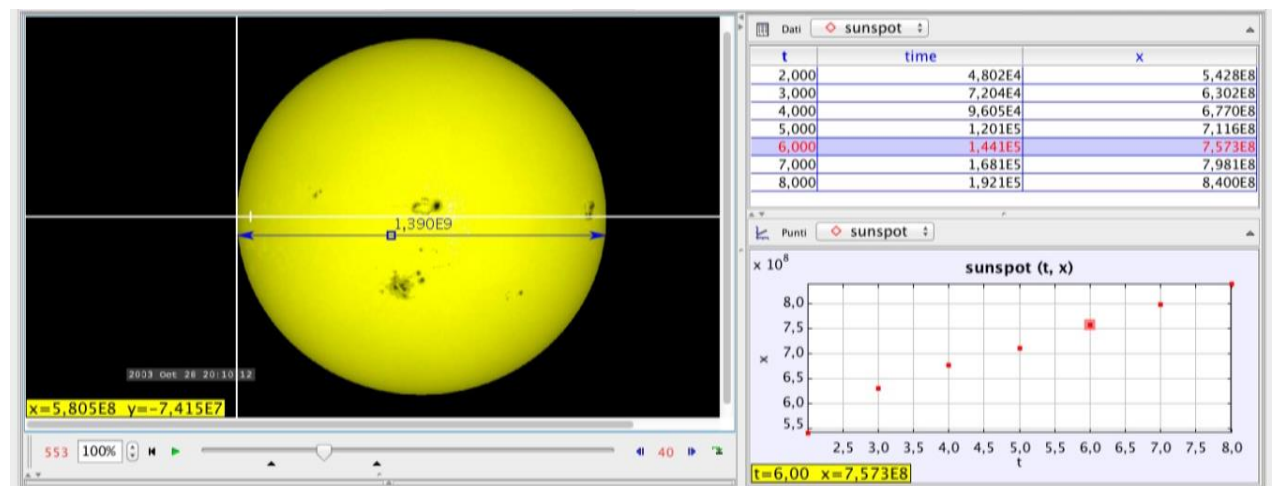

Fig 3. Tracker analysis of a video representing the motion of sunspots at equatorial latitude (https://svs.gsfc.nasa.gov/3502) during a two-week period in October and November of 2003, obtained with Michelson Doppler Interferometer (MDI). Left frame shows the tracking of the sunspot, right frame shows position vs. time graph and table of the sunspot. A linear fit gives an estimate of the Sun rotational velocity $v=(1.9 \pm 0.1) 10^{3} \mathrm{~ms}^{-1}$.

5 Data used are from http://va-iitk.vlabs.ac.in/data/expt4/solar_data.txt. Note that data have been not corrected for instrumental profile response. 


\subsection{Fourth phase: energy and nuclear reactions}

The driving question used to introduce the last phase of our TM is: "How do you think a star is functioning?" Students are familiarized with the stars' inner mechanism that accounts for pressure forces. We ask students to hypothesise which kind of process could allow the Sun to emit energy up from its birth to nowadays. Burning processes are first reviewed, as they are the most simple and energetic reactions available in everyday life. Students are encouraged to estimate how much energy it is possible to obtain from burning a mass of $H$, oil, wood and other burning materials, equivalent to that of the Sun. Knowing the actual solar power, students are also encouraged to estimate how long the Sun could live emitting this amount of energy if its source was only a burning reaction involving the burning materials examined. A simple calculation leads to a maximum time of about 23000 years, which evidently is too small a value for the age of the Sun. We then introduce the $H$ $\rightarrow \mathrm{He}^{4}$ production to reinforce the idea that stellar processes consist of nuclear reactions that produce photons and thermal energy, which are responsible for pressure forces that contrast gravitational force. In particular, we show that mechanical and thermal equilibrium conditions hold until the nuclear reactions processes are efficient enough to oppose gravitational effects.

\section{Implementation and evaluation of the module}

\subsection{Sample}

The module was piloted with two intact classes of secondary school students $(N=59$ students, 17-18 years old) involved in extra-curricular activities organized by the Department of Physics of the University of Naples "Federico II". The total duration followed that of Table 1 (about 20 hours). To assess the module's effectiveness on students' understanding about stars, we designed a ten-question multiple-choice instrument adapted from two existing instruments $[5,13]$. The questionnaire, available in Colantonio et al. [14], was submitted before and after the activities. We assess students' answers giving 1 or 0 point according to whether they were correct or not. Table 2 shows the correspondence between the questionnaire's items and the module's key ideas.

Table 2: Description of the items of the questionnaire

\begin{tabular}{|c|c|c|}
\hline $\mathrm{N}$ & Question & Key idea \\
\hline 1 & What is a star? & Mechanical and thermal equilibrium \\
\hline 2 & How do you think a star is formed? & Mechanical and thermal equilibrium \\
\hline 3 & What are the main stellar inner processes? & Energy and nuclear reactions \\
\hline 4 & $\begin{array}{l}\text { What are the forces involved in the process of stellar } \\
\text { formation? }\end{array}$ & Mechanical and thermal equilibrium \\
\hline 5 & What influences the shape of a star? & Mechanical and thermal equilibrium \\
\hline 6 & What factor does the temperature of a star depend on? & Energy and nuclear reactions \\
\hline 7 & $\begin{array}{l}\text { (Four drawings are shown) Which drawing represents the } \\
\text { process by which an absorption line is formed? }\end{array}$ & Spectral analysis \\
\hline 8 & Coolest stars emit most of their energy in...? & Spectral analysis \\
\hline 9 & What happens during the evolution of a star? & Energy and nuclear reactions \\
\hline 10 & $\begin{array}{l}\text { (Three spectral curves are shown) Which of the objects has } \\
\text { the highest temperature? }\end{array}$ & Spectral analysis \\
\hline
\end{tabular}

\subsection{Results}


Analysis of students' outcomes is reported in Fig. 4 and 5. Average pre-test score was 1.90 \pm 0.14 (st. err.), while that of the post-test was $5.3 \pm 0.3$ (st. err.). The difference is statistically significant $\left(t=11.420, d f=87.849, p<10^{-4}\right)$. Average percentage of correct answers in the pre- and post-test for the three key ideas is reported in Table 3. In the pretest, students found many difficulties in answering questions about nuclear reactions in stars: for instance, only $8 \%$ of the students answered correctly about the evolution of the star. Some difficulties emerged in answering questions about forces involved in a star's formation and shape. Finally, students confused the processes of energy absorption and emission in atoms. In the post-test, students show better results. Concerning mechanical and thermal equilibrium, majority of students (about 70\%) were able to recognize correctly that a star is a structure in gravitational equilibrium and the role of gravitational force in the collapse of a nebula. It can be plausibly inferred that the proposed tasks on gravity enhanced students' understanding of this key idea. Concerning spectral analysis, the relationship between temperature and emitted wavelength seems to have been understood by students. We still detected some problems concerning mechanisms of emission and absorption of light, evidence that suggests improving activities to better relate microscopic models of atoms to stars' light emission. Finally, concerning energy and nuclear reactions, the proposed tasks appear to be effective in improving students' ability to distinguish between nuclear and chemical reactions. However, the consequences of these reactions often remain confused. This evidence suggests revising the proposed tasks to provide students more opportunities to deepen their knowledge about nuclear reactions.

Table 3: Average percentages of correct answers grouped according to module's key ideas.

\begin{tabular}{lcc}
\hline Key Ideas & Pre (\%) & Post (\%) \\
\hline Energy and nuclear reactions & 14 & 40 \\
Mechanical and thermal equilibrium & 25 & 66 \\
Spectral analysis & 14 & 56 \\
\hline
\end{tabular}

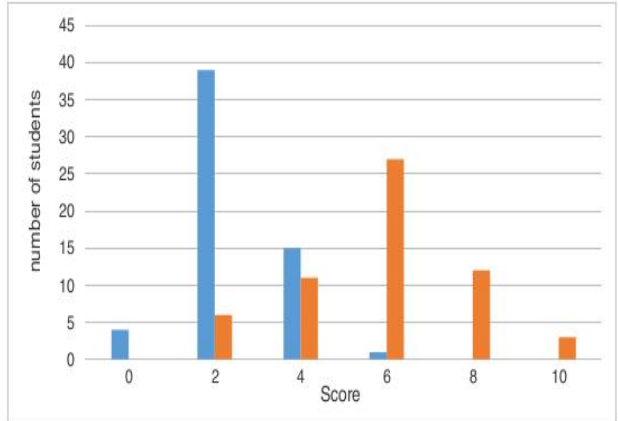

Fig. 4. Students' score distribution in the pre- and post-activity questionnaire

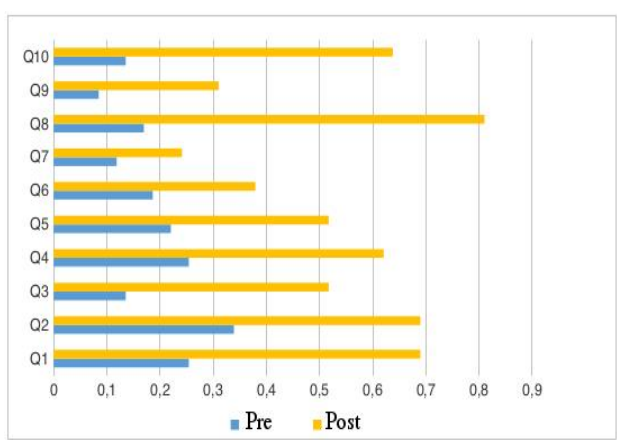

Fig. 5. Percentage of students' correct answers in the pre- and post-activity questionnaire

\section{Conclusions}

In this paper, we have presented an innovative module that aims at improving secondary school students' knowledge about stars focusing on three key ideas: mechanical and 
thermal equilibrium, spectral analysis, energy and nuclear reactions. Throughout the proposed activities, we mainly focus students' attention on experiments, laws and models that bring together different content areas, with the aim of avoiding the risk of transmitting a fragmented view of physics.

The analysis of students' outcomes before and after the teaching intervention supports the effectiveness of the proposed activities in understanding basic concepts about stars and mechanisms underlying their functioning, in particular the mechanical equilibrium and the relationships between surface temperature and emitted wavelength. Plausibly, by blending practical tasks involving classical physics laws and laboratory/computer-based activities to study light spectra and the motion of the Sun, students have improved their knowledge about these specific aspects of stars. Consistent with literature findings, before the activities most students had scarce knowledge about the nuclear fusion processes happening in a star's core; after the activities, the percentage of students who at least recognized nuclear reactions as relevant for stars' functioning increases up to $50 \%$. Plausibly, the quantitative task of comparing the energy delivered by an equivalent mass of $H$ and the actual energy provided by the Sun may have helped students to overcome the "burning" misconception [7]. Moreover, our TM addresses students' difficulty in justifying the existence in the Universe of elements heavier than $H$ and $H e$ and improves their knowledge of element production mechanisms. Finally, the proposed activities on star formation and evolution may help students to acquire a first insight into anisotropy and non-homogeneity of the Universe at small scale.

Overall, hence, our study supports the use of stars as rich and motivating context to help students achieve high-level, "expert", scientific reasoning skills. As a future step of this study, we are planning to enrich our TM by including tasks about the birth, evolution, and death of stars by deepening the role of the star's mass.

\section{References}

1. M. Barnett and J. Morran, Addressing children's alternative frameworks of the Moon's phases and eclipses, International Journal of Science Education, 24 859-879 (2002)

2. J.D. Plummer, C. Palma, A. Flarend, K. Rubin, Y. Shiou Ong, B. Botzer, S. McDonald and T. Furman, Development of a Learning Progression for the Formation of the Solar System, International Journal of Science Education 37 1381-1401 (2015)

3. J. D. Plummer and L. Maynard, Building a learning progression for celestial motion: An exploration of students' reasoning about the seasons, Journal of Research in Science Teaching 51 902-929 (2014)

4. I. Testa, G. Busarello, E. Puddu, S. Leccia, P. Merluzzi, A. Colantonio, M. I. Moretti, S. Galano, A. Zappia, Quantitative experiments to explain the change of Seasons, Physics Education 50 179-188, (2015)

5. J. M. Bailey, B. Johnson, E. E. Prather and T.F. Slater, Development and Validation of the Star Properties Concept Inventory International Journal of Science Education 34 14 2257-2286 (2012)

6. M. Finegold and D. Pundak, Students' conceptual frameworks in astronomy, Australian Science Teachers Journal 36 76-83 (1990)

7. L. Agan, Stellar ideas: Exploring students' understanding of stars, Astronomy Education Review 3(1) 77-97 (2004) 
8. J. M. Bailey, Development of a concept inventory to assess students' understanding and reasoning difficulties about the properties and formation of stars ( $\mathrm{PhD}$ dissertation), The University of Arizona, Tucson, AZ, USA (2006)

9. J. M. Bailey, E. E. Prather, B. Johnson and T. F. Slater, College Students' Preinstructional Ideas About Stars and Star Formation, Astronomy Education Review 81 (2009)

10. S. Leccia, A. Colantonio, E. Puddu, S. Galano and I. Testa, Teaching about mechanical waves and sound with a tuning fork and the Sun, Physics Education, 50 677-689 (2015)

11. J. Persson, Sunspots and solar rotation, Physics Education, 48 14-6 (2013)

12. C. I. Ribeiro, Sunspots and their simple harmonic motion, Physics Education, 48 586588 (2013)

13. E. M. Bardar, E. Edward, E.E. Prather, K. Brecher and T. F. Slater, Development and Validation of the Light and Spectroscopy Concept Inventory, Astronomy Education Review, 25 103-113 (2007)

14. A. Colantonio, S. Galano, S. Leccia, E. Puddu and I. Testa (2017), A teaching module about stellar structure and evolution. Physics Education, Vol.52, n 1, 015012, doi http://dx.doi.org/10.1088/1361-6552/52/1/015012 